\title{
Temperature evolution and grain defect formation during single crystal solidification of a blade cluster
}

\author{
${ }^{*}$ De-xin Ma ${ }^{1,2}$, Fu Wang ${ }^{2}$, Qiang Wu ${ }^{3}$, Jian-zheng Guo ${ }^{1}$, Fu-ze Xu' ${ }^{1}$, Zhao-feng Liu', and Shou-zhu Ou ${ }^{1}$ \\ 1. Wedge Central South Research Institute, Shenzhen 518045, China \\ 2. Foundry Institute, RWTH Aachen University, Aachen 52072, Germany \\ 3. ALD Vacuum Technologies GmbH, Hanau 63450, Germany
}

\begin{abstract}
In order to investigate the asymmetry of thermal conditions during directional solidification, the temperature evolution and correspondingly developed undercooling in a simplified single crystal blade cluster were numerically simulated. Simulation results demonstrate that the temperature distribution at the blade platforms is obviously asymmetrical. On the outside of the blade which directly faces the heating element, the liquidus $\left(T_{L}\right)$ isotherms progress relatively smoothly. On the inside of the blades facing the central rod, however, the $T_{L}$ isotherms are in concave shape and the slope goes upwards to the platform extremities. The average undercooling extent $\Delta T$ and undercooling time $\Delta t$ at the inside are much higher than those at the outside. It was then predicted that the inside platform extremities have significantly higher probabilities of stray grain formation compared to the outside ones. A corresponding experiment was carried out and the metallographic examination exhibited the same side- and height-dependence of stray grain formation in the blades as predicted. On the inside of the blades, all platforms are occupied by stray grains, while the platforms on the outside are nearly stray grain free. The simulation result agrees very well with the experimental observation.
\end{abstract}

Key words: superalloy; directional solidification; single crystal (SC); simulation; stray grain (SG)

CLC numbers: TP391.99 Document code: A Article ID: 1672-6421(2017)05-456-05

Cingle crystal (SC) solidification of turbine blades for advanced gas turbines is a key technology for the production of reliable and high efficiency gas turbines. The SC blades of superalloys are currently produced by using investment casting combined with the well-known Bridgman process, which utilizes the concept of mold translation and a radiation baffle to sharpen the thermal gradient. In the production of SC blades for aeroengines, the blades are usually assembled into cluster configuration, which enables several parts to be produced in a single casting cluster. In a conventional Bridgman furnace, the heater and the cooler have a cylindrical construction. Correspondingly, a shell mold cluster for a group of parts is arranged in a circle around a central rod.

\section{*De-xin Ma}

Male, born in 1955, Ph.D., Professor. His research mainly focuses on investment casting and single crystal solidification of superalloy turbine blades. He has published more than 110 papers.

E-mail: d.ma@gi.rwth-aachen.de

Received: 2017-08-15; Accepted: 2017-09-27
During SC solidification in turbine blades of superalloys, grain defects often form in the platform region with abrupt variation in cross-section. It is known that stray grains in $\mathrm{SC}$ parts are related to curvature of the liquidus isotherm while it is passing through enlargements in the cross-section of the component (e.g. the platform of a turbine blade) ${ }^{[1,2]}$. As the solidification front approaches the platform, a significant degree of undercooling develops in the liquid at the extremity. When the undercooling exceeds the undercoolability of the used alloy, new grains may nucleate to form macroscopic stray grain (SG) defects ${ }^{[3-5]}$.

The prevention of grain defect formation in modern $\mathrm{SC}$ turbine blade manufacturing is increasingly challenging. The effects of various casting conditions on grain defects have been investigated and some progresses have been achieved in experiments and simulations ${ }^{[3-8]}$. However, the SG formation in largescale production with casting clusters of multiple blades has not been sufficiently investigated, due to the complicated solidification process. In this study, the asymmetrical thermal condition in a casting cluster was 
systematically examined by using temperature simulation. The prediction of grain defect formation during the SC solidification was compared with the experimental results.

\section{Design of blade geometry and cluster arrangement}

A simplified turbine blade geometry was designed for simulation

(a)

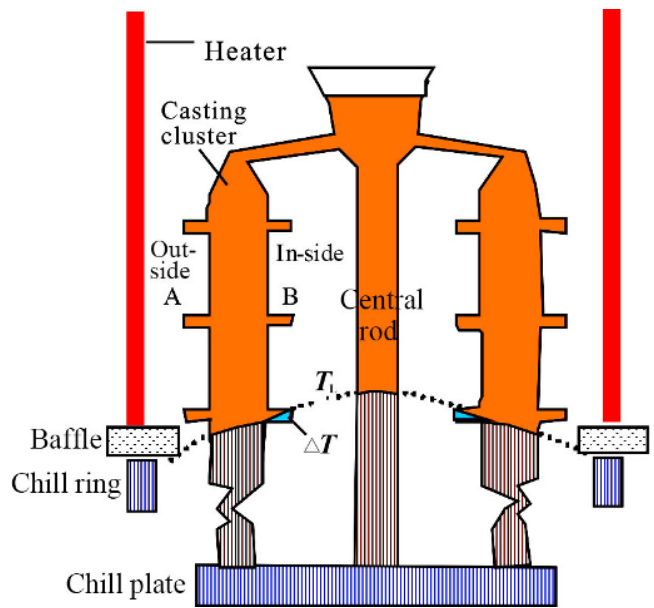

and experiment. It consists of a plate of $5 \mathrm{~mm}$ in thickness, 30 $\mathrm{mm}$ in width and $150 \mathrm{~mm}$ in height as blade body. At platform sections, a number of protrusions extend $10 \mathrm{~mm}$ on both sides from the blade body. Usually, there are one or two platforms in the turbine blades. In this work, however, three steps of platforms are designed in each blade to investigate the SG formation at different heights (bottom, middle and top) of the blades (Fig. 1a).

Fig. 1: Geometry of simplified turbine blades and schematic of Bridgman process for SC blade cluster (a) Side view; (b) Top view

According to the cylindrical construction of the Bridgmantype furnace, 5 blades were assembled in a cluster around a central rod (Fig. 1b). The outside of the cluster (A-side) can be immediately irradiated by the heater, providing a favorable thermal condition for SC growth. In contrast, the heat exchange on the inward side (B-side) facing central rod is inefficient, leading to the formation of grain defects. This assumption should be confirmed by simulation and experiment in this work.

\section{Simulation and experimental procedures}

The computing equipment in the present work is a SGI Workstation. In the pre-process, the digital description of the furnace and casting with shell mold and also the subsequent FE mesh generation were executed using the commercial software IDEAS supertab. The appropriate material properties for Ni-based superalloys and a ceramic mold $\left(\mathrm{Al}_{2} \mathrm{O}_{3}\right)$ were entered into the computer, including the heat conductivity and the heat capacity as functions of temperature. The alloy used in this investigation is a Ni-based superalloy IN 939 SC (Cr 22.4, Co 19.0, W 2.0, Ta 1.3, Al 1.9, Ti 3.7 and $\mathrm{Nb} 1.0$ in wt.\%) modified for the production of SC blades. The liquidus and solidus temperatures of the used alloy, and $T_{\mathrm{S}}$, were previously measured to be $T_{\mathrm{L}}=1326{ }^{\circ} \mathrm{C}$ and
$T_{\mathrm{S}}=1274{ }^{\circ} \mathrm{C}$ respectively, revealing the freezing range $\left(T_{\mathrm{L}}-T_{\mathrm{S}}\right)$ of $52{ }^{\circ} \mathrm{C}$. The fraction of solid was calculated using the Scheil model to obtain the temperature dependent release of latent heat within the freezing range. The macroscopic solidification modeling was executed using a computer software called CASTS (Computer Aided Solidification Technologies) for simulation of the Bridgman process to fully describe the heat transfer balance in the vacuum furnace, including calculation of view factor radiation.

The simulation was performed for the same condition as designed for a running experiment. The process parameters, such as the furnace temperature of $1,500{ }^{\circ} \mathrm{C}$ and the $3.0 \mathrm{~mm} \cdot \mathrm{min}^{-1}$ withdrawal velocity of the mold from the hot zone to the cold zone, were inputted as the cast process conditions. During the calculation of the transient temperature evolution, the release of latent heat within the freezing range was taken into account. It took about four hours to calculate the position dependent viewing factors. The three dimensional (3D) simulation of solidification process of a blade cluster could be finished within one hour. The macroscopic shape and progress of the liquidus isotherm were visualized in the post-process.

The casting experiment was performed according to the standard Bridgman process. The ceramic shell mold was produced by an investment casting procedure. After the assembly of the wax cluster, the shell mold was made by repeating the 
procedure of dipping in ceramic slurries and sanding with fused alumina. After dewaxing in a steam autoclave, the shell mold was fired at $1,200{ }^{\circ} \mathrm{C}$ in a firing furnace. The shell mold was finally placed on the chill plate in the Bridgman furnace for casting. After preheating the shell mold and melting the alloy ingot in crucible, the liquid metal was poured into the shell mold. The mold in the furnace was then withdrawn from the hot zone to the cold zone through the baffle at the designed velocity of $3.0 \mathrm{~mm} \cdot \mathrm{min}^{-1}$

\section{Results and discussion}

\subsection{Temperature simulation}

As stated above, numerical simulation was performed ahead of the casting experiments. Figure 2 exhibits the simulated temperature evolution for 3 steps of platforms in different heights of the blade. Besides the temperature variation during the withdrawal process, the most distinct features are the nonuniformity of the temperature distribution in the geometrically symmetric blades. For all 3 platform steps (bottom, middle and top), the temperature changes not only vertically, but also horizontally between the outside (A) and the inside (B). Considering the radiation between the furnace heater and the shell mold, the asymmetry of the thermal condition in blades is mainly caused by a unilateral heating of each single blade. The outside A (also called heater-side) of a blade is immediately illuminated and heated by the heater. In contrast, a direct heater radiation on the inside $\mathrm{B}$ (also called shadow-side) is impossible due to the shielding by the central rod and other components in the cluster. This shadow side can hence be ineffectively heated, leading to the obviously poor thermal exchange for SC growth.

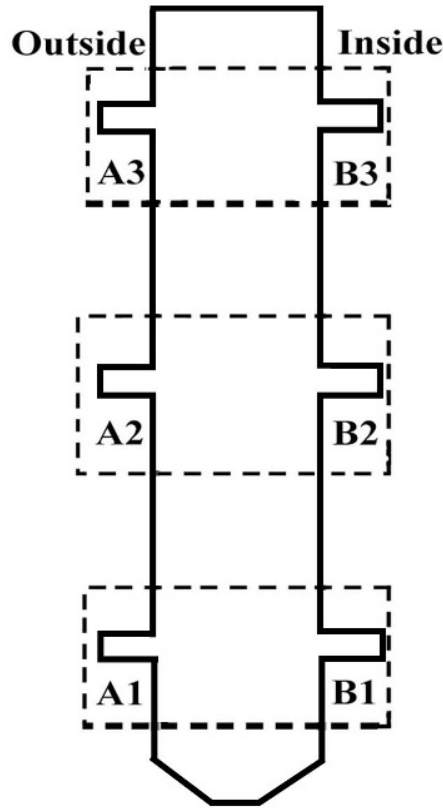

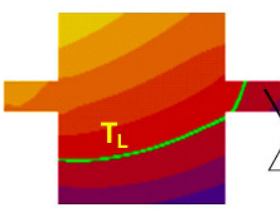

$3925 \mathrm{~s}$

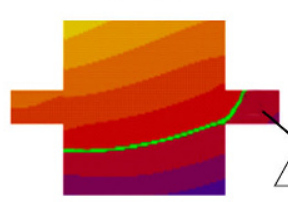

$2975 \mathrm{~s}$

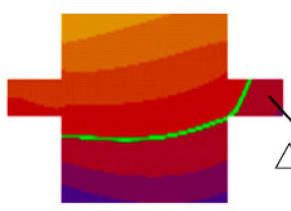

$2000 \mathrm{~s}$

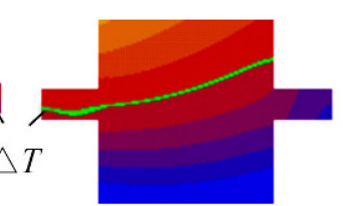

$4125 \mathrm{~s}$

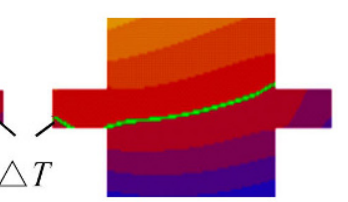

$3000 \mathrm{~s}$

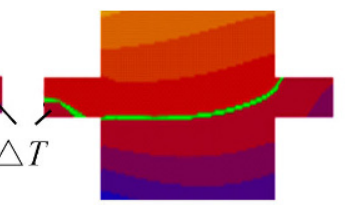

$2050 \mathrm{~s}$

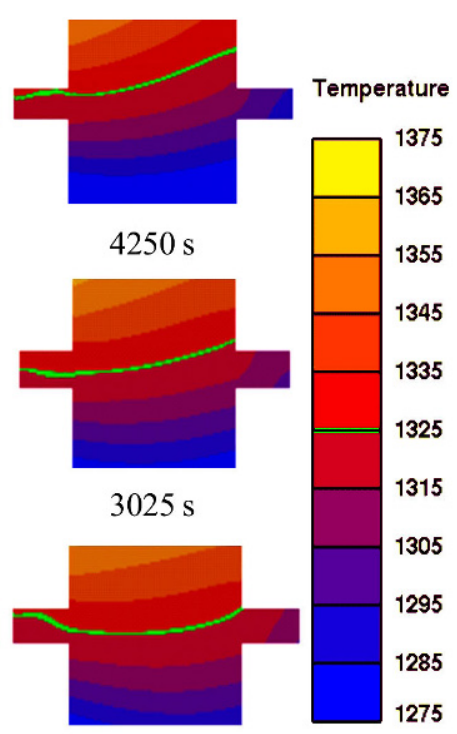

$2075 \mathrm{~s}$

Fig. 2: Simulated $T_{\mathrm{L}}$-development at different heights, indicating melt undercooling $(\Delta T)$ on platforms

As shown in Fig. 2, the liquidus $\left(T_{\mathrm{L}}\right)$ isotherms in the blades are concave. Especially on the inside B facing the central rod, a relatively deeply undercooled zone is formed at the extremity before the solidification front advances slowly into the platform, where nucleation of a new grain is almost unavoidable. On the A side, the liquidus isotherms were much smoother, leading to much lower undercooling $\Delta T$. Before the $T_{\mathrm{L}}$-isotherm in the blade body reaches the platform, the undercooled zone at the platform extremities remains thermally isolated for a time $\Delta t$, over which local undercooling continues to increase. New grains would nucleate to generate macroscopic SG defects if the local undercooling $\Delta T$ was sufficient to exceed the critical undercooling for nucleation, namely undercoolability ${ }^{[8]}$. The probability of SG nucleation at platform extremities is then directly dependent on both the local undercooling extent $\Delta T$ and the whole undercooling time $\Delta t$.

Based on the temperature simulation, the local undercooling extent $\Delta T$ and the undercooling duration $\Delta t$ at different platform extremities of the investigated blade were evaluated and listed in Table 1, indicating their position dependence in the blade cluster. On the $\mathrm{B}$ side, the average value of $\Delta T$ and $\Delta t$ were calculated to be about $15 \mathrm{~K}$ and $150 \mathrm{~s}$, respectively. On the A side, they were reduced significantly to $\Delta T=$ $4.6 \mathrm{~K}$ and $\Delta t=21.9 \mathrm{~s}$. Due to the lower undercooling and shorter duration, the probability of SG nucleation on the A side must be significantly smaller than that on the B side, as experimentally confirmed in the next section.

Figure 3 graphically shows the simulated undercooling extent 
Table 1: Simulated undercooling extent $\Delta T$ and time $\Delta t$ at different sides and heights of blade

Outside (A)
$\Delta T(\mathrm{~K})$

of $\Delta T$ and $\Delta t$ at different platforms. The position dependence of the SG formation probability can then be ranked as: $\mathrm{B} 3>\mathrm{B} 2>\mathrm{B} 1>\mathrm{A} 1>$ $\mathrm{A} 2>\mathrm{A} 3$. As predicted in Fig. 3, the $\mathrm{B}$ side is very prone to SG formation, especially at the highest platform (B3), followed by the middle (B2) and bottom one (B1). In comparison, the A-side has much less tendency to SG formation. It should be noted that higher platforms (A3 and A2) have even less SG forming tendency than the lowest one (A1), indicating a contrary height dependence of SG formation to the B side.

\subsection{Experimental examination}

After the Bridgman process, the blades were shaken out from the mold and macroscopically etched to reveal the grain and dendrite structure at the surface. Figure 4 shows the surface macrostructure of 5 blades whose left and right sides represent the outside (A) and inside (B) in the casting cluster, respectively. The observed stray grains are marked with frames. As shown in Fig. 4, the metallographic examination exhibited a significant difference in the SG formation on different platform halves. On the Side B facing the central rod, all 15 platforms contained stray grains, revealing a $\mathrm{SG}$ ratio of $Q_{\mathrm{N}}=15 / 15=1$. On Side A facing the heater, only 2 platforms in 2 blades (No. 2 and No. 4) are partly occupied by $\mathrm{SG}$, exhibiting a SG ratio of $Q_{\mathrm{N}}=2 / 15=0.13$. This shows clearly that the B-Side of the blade platforms has a markedly higher tendency for SG formation.

As a typical feature, Figure 5 shows the longitudinal sections of the platforms in a blade (No. 4 in Fig. 4), in which the SG structure can be observed in more detail. It is clearly observed that platforms A2 and A3 are free of SG, while platforms A1, B1, B2 and B3 feature increasing portions of SG.
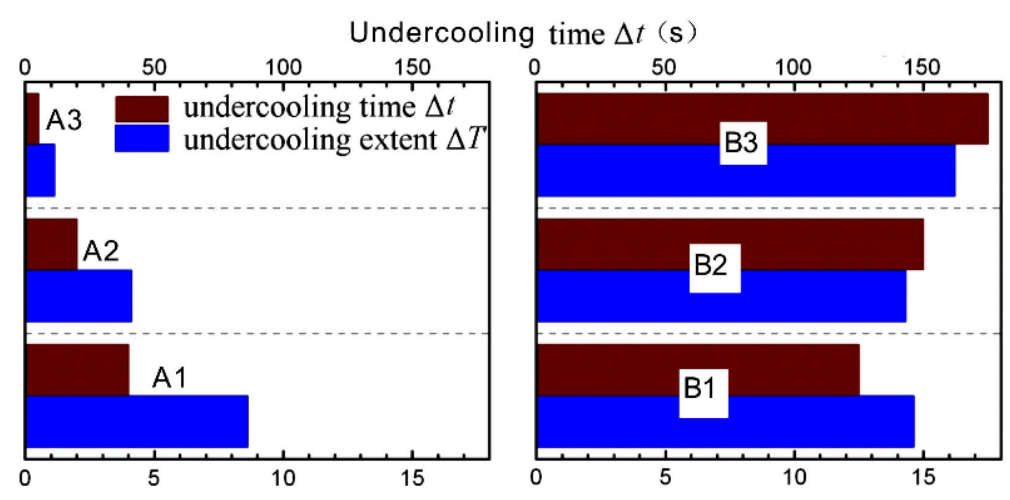

(a)

Undercooling extent $\Delta T(\mathrm{~K})$

(b)

Fig. 3: Simulated undercooling extent $\Delta T$ and time $\Delta t$ in blade platforms on A-side (a) and B-side (b)

In order to evaluate the extent of SG formation quantitatively, the volume ratio $Q_{\mathrm{V}}$ of $\mathrm{SG}$ is defined, which indicates the $\mathrm{SG}$ volume in proportion of the platform. The volume is calculated by the coverage of stray grains on the platform. This $Q_{\mathrm{V}}$ is a practical indicator of the consequence of competitive growth between stray grains starting from the extremity and primary crystals from the blade body. A value of $Q_{\mathrm{V}}=1$ means total occupation by stray grains, while a value of $Q_{\mathrm{V}}=0$ indicates a platform completely free of stray grains. All 15 platform halves on the inside and outside were respectively

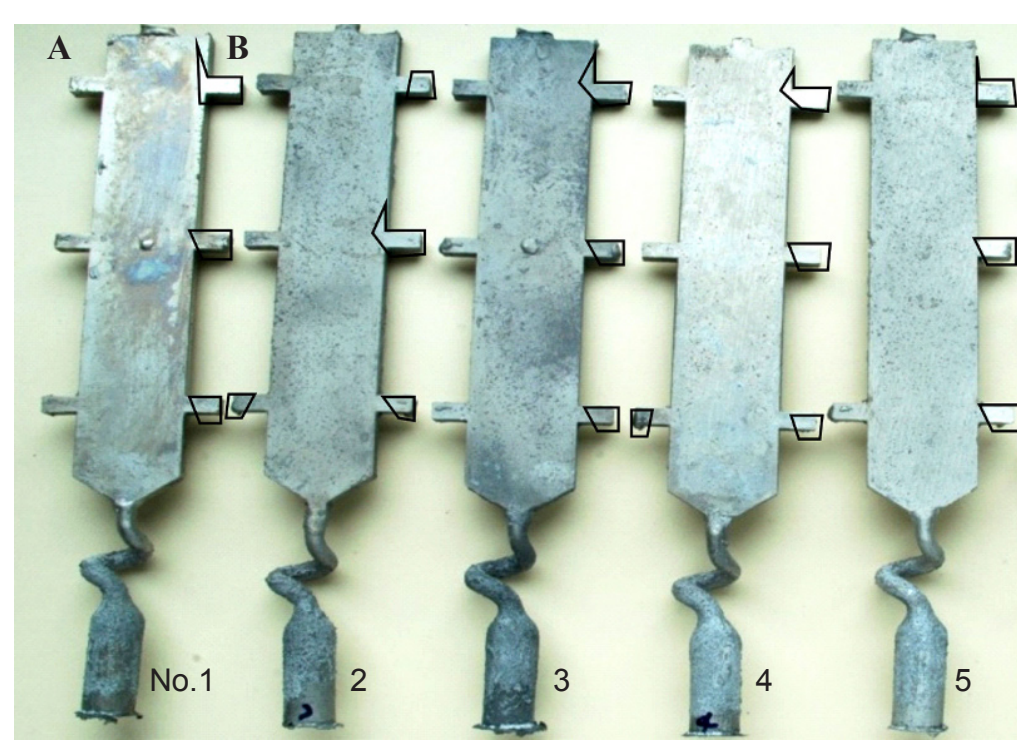

Fig. 4: Five blades solidified in a cluster, revealing SG formation on platforms, denoted by frames 
examined. The average $Q_{\mathrm{V}}$ values for each platform of the blades and their mean value on each step and each side are listed in Table 2.

According to the experimental examinations shown in Table 2, and Figs. 4 and 5, the most distinct features of the results can be concluded as follows:

(1) The B-side is significantly prone to SG formation compared to A-side, since the mean $Q_{\mathrm{V}^{-}}$values of the two sides are 0.83 and 0.04 , respectively. This experimentally determined side-dependence conforms the prediction (Table 1 and Fig. 3), which shows a higher undercooling extent $\Delta T$ and longer undercooling time $\Delta t$ on the $\mathrm{B}$-side than on the A-side.

(2) On the A-side of the investigated blades, the mean value of $Q_{\mathrm{V}}$ at the bottom platform (A1) is 0.13 , revealing a higher SG forming tendency than the middle (A2) and top (A3) ones $\left(Q_{\mathrm{V}}\right.$ $=0)$. This is also in good agreement with the simulation results (Table 1 and Fig. 3)

(3) On the B-side, however, all top platforms (B3) are completely occupied by stray grains $\left(Q_{\mathrm{V}}=1\right)$, revealing the

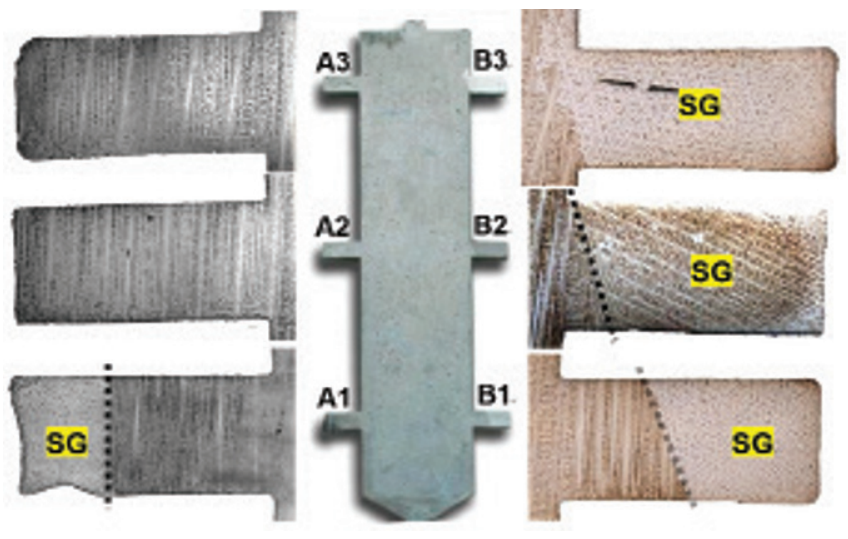

Fig. 5: Longitudinal sections of platforms in blade 4 (see Fig. 4), showing SG formation in more detail

highest SG forming tendency than the middle $\left(\mathrm{B} 2: Q_{\mathrm{V}}=0.85\right)$ and bottom one (B1: $\left.Q_{\mathrm{V}}=0.65\right)$. This height dependence of SG formation is contrary to that on the A-Side, just as the simulation predicted.

Table 2: Average values of $Q_{N}$ and $Q_{V}$ on the platforms in different sides and heights, evaluated from 5 blades in Fig. 5.

\begin{tabular}{|c|c|c|c|c|c|}
\hline$Q_{N}$ & $\boldsymbol{Q}_{\mathrm{v}}$ & A-side & B-side & $\mathbf{Q}_{\mathrm{N}}$ & $Q_{v}$ \\
\hline $0 / 5$ & 0 & $\mathrm{~A} 3-$ & B3 & $5 / 5$ & 1 \\
\hline $0 / 5$ & 0 & A2- & B2 & $5 / 5$ & 0.85 \\
\hline $2 / 5$ & 0.13 & $\mathrm{~A} 1$ & B1 & $5 / 5$ & 0.65 \\
\hline 0.13 & 0.04 & mean & mean & 1 & 0.83 \\
\hline
\end{tabular}

\section{Conclusions}

The thermal conditions and the corresponding grain defect formation in the Bridgman process of a SC blade cluster were investigated through simulation and experiment. The simulated temperature distribution shows a more concave curved liquidus $\left(T_{\mathrm{L}}\right)$ isotherm on the inside of the blade, with the average undercooling extent $\Delta T$ and undercooling time $\Delta t$ much higher than those on the outside. It was then predicted that the inside platform extremities have significantly higher probabilities of SG formation compared to the outside ones. A corresponding experiment was carried out with superalloy IN 939 SC. The metallographic examination exhibits the same side- and height-dependence of SG formation in the blades as predicted. On the inside of the blades, all platforms are occupied by stray grains, while the platforms on the outside are nearly SG free. The simulation result agrees very well with the experimental observation.

\section{References}

[1] Paul U, Sahm P, Goldschmidt D. Inhomogeneities in single-crystal components. Mat. Sci. Eng. A, 1993, 173: 49-54.

[2] Meyer ter Vehn M, Dedecke D, Paul U, et al. Undercooling related casting defects in single crystal turbine blades. R. Kissmger, D. Deye, D. Anton, et al, eds., Superalloys 1996, TMS, Warrendale, PA, 1996: 471-479.

[3] Ma D, Bührig-Polaczek A. Development of heat-conductor technique for single crystal components of superalloys. Int. J. Cast Met. Res., 2009, 22: 422-429.

[4] Ma D, Bührig-Polaczek A. Application of heat-conductor technique to production of single crystal turbine blade. Metall. Mater. Trans. B, 2009, 40: 738-748.

[5] Ma D, Wang F, Wu Q, et al. Innovation of casting techniques for single crystal turbine blades of superalloys. M. Hardy, E. Huron, U. Glatzel, et al, eds., Superalloys 2016, TMS, Warrendale, PA, 2016: 237-246.

[6] Napolitano R, Schaefer R. The convergence-fault mechanism for low-angle boundary formation in single-crystal castings. J. Mater. Sci., 2000, 35: 1641-1659.

[7] $\mathrm{Yu} \mathrm{J,} \mathrm{Xu} \mathrm{Q} \mathrm{Y,} \mathrm{Cui} \mathrm{K,} \mathrm{et} \mathrm{al.} \mathrm{Numerical} \mathrm{simulation} \mathrm{of} \mathrm{solidification}$ process on single crystal $\mathrm{Ni}$-based superalloy investment castings. J. Mater. Sci. Technol., 2007, 23: 47-54.

[8] Ma D, Wu Q, Bührig-Polaczek A. Investigation on the asymmetry of thermal condition and grain defect formation in customary directional solidification process. IOP Conf. Series: Mat. Sci. Eng., 2011, 27: 012037

This work was financially supported by the "Shenzhen Peacock Plan" and the "Guangdong Innovative \& Entrepreneurial Research Team Program". 